\title{
Thermoelectric Properties of n-type Bismuth Telluride Based Alloys Prepared by Hot Pressing and Zone Melting Method
}

\author{
Heon Phil HA*, Young Joo OH*, Dow Bin HYUN* and Eui Pak Yoon** \\ *Metals Processing Center, Korea Institute of Science and TechnologyP.O. box 131, Cheongryang, Seoul, Korea \\ *:Department of Metallurgical Engineering, Hanyang University, 17 Heangdangdong, Seongdong, Seoul, Korea \\ E-mail :Heonphil@kist.re.kr
}

\begin{abstract}
Alloys of $\mathrm{Bi}_{2} \mathrm{Te}_{3}$ rich side of $\mathrm{Bi}_{2} \mathrm{Te}_{3}-\mathrm{Bi}_{2} \mathrm{Se}_{3}$ were prepared by the zone melting method and the hot pressing method in order to compare their thermoelectric properties. When specimens were hot pressed thermoelectric properties changed as a function of particle size, pressing time and hot pressing temperature. The reasons for the variation of the thermoelectric properties were investigated by examining influences of following parameters; oxidation, mechanical deformation during pulverization and the hot pressing temperature. Thermoelectric properties of zone melted ingot were largely affected by dopants when composition is fixed, whereas thermoelectric properties of hot pressed material were mainly related with the variation of the carrier concentrations caused by the generation of electrically active defects from many sources. Defects induced by the mechanical deformation and oxygen causes generation of donors. Defect concentration is also altered with different hot pressing temperatures depending on the amount of previously received mechanical deformation.
\end{abstract}

Key Word : $\mathrm{Bi}_{2} \mathrm{Te}_{3}-\mathrm{Bi}_{2} \mathrm{Se}_{3}$ alloy, hot pressing, zone melting, carrier generation, mechanical deformation.

\section{Introduction}

Bismuth telluride based solid solutions have been widely used as materials for thermoelectric energy conversion near room temperature. Modules are composed of p-type $\mathrm{Bi}_{2} \mathrm{Te}_{3}-\mathrm{Sb}_{2} \mathrm{Te}_{3}$ and n-type $\mathrm{Bi}_{2} \mathrm{Te}_{3}-\mathrm{Bi}_{2} \mathrm{Se}_{3}$ elements. Specially, thermoelectric modules are effective for applications with small cooling capacity like electronic parts. In ordinary, these materials are grown by a directional solidification method to use excellent thermoelectric properties to the growth direction. Materials grown by a directional solidification method, however, have a disadvantage of poor mechanical properties because the basal plane in this rhombohedral structure is cleavage plane. Currently, elements with robust mechanical property are required in the response to an increased demand for micro modules. One of methods to enhance the mechanical properties is to use the powder metallurgy. There have been quite a lot of studies for the n-type materials [1-3]. However, the difference of carrier generation mechanisms in two different growth methods was not clarified. In the present study, mechanisms of carrier generations during hot pressing and zone melting process of the n-type $\mathrm{Bi}_{2} \mathrm{Te}_{3}-\mathrm{Bi}_{2} \mathrm{Se}_{3}$ system were investigated because thermoelectric properties are largely affected by the amount of the carrier concentration when the composition of alloy is fixed. In the preliminary study of hot pressing of the $\mathrm{Bi}_{2} \mathrm{Te}_{3}-\mathrm{Bi}_{2} \mathrm{Se}_{3}$ system, it was found that thermoelectric properties changed drastically depending on process parameters. Studies carried out for the directional growth method could not explain the changes. Detailed studies have been carried out on effects of the mechanical deformation, oxidation and hot pressing parameters to the carrier generation.

\section{Experimental procedure}

High purity $(99.99 \%) \mathrm{Bi}, \mathrm{Te}$, and Se granules $(\sim 5 \mathrm{~mm})$ were washed with $10 \%$ nitric acid, acetone, and distilled water to remove surface oxide layers. Appropriate amounts of $\mathrm{Bi}, \mathrm{Te}$ and Se were weighed to make $250 \mathrm{~g}$ of $\mathrm{Bi}_{2} \mathrm{Te}_{3}-\mathrm{Bi}_{2} \mathrm{Se}_{3}$ solid solutions and charged into a quartz tube. The inside wall of the quartz tube was carbon-coated by the acetone cracking. The quartz tube was evacuated to $10^{-5}$ torr and sealed. $\mathrm{Bi}, \mathrm{Te}$ and $\mathrm{Se}$ in the quartz tube were melted at $800^{\circ} \mathrm{C}$ for 2 hours using a rocking furnace to ensure composition homogeneity, and quenched to room temperature. For the zone melting growth quenched ingot was directionally grown at $0.1 / \mathrm{min}$. The furnace temperature was $700^{\circ} \mathrm{C}$. For the hot pressing, ingots were pulverized and sieved into different sizes of $\sim 38,38 \sim 90$ and $90 \sim 250 \mu \mathrm{m}$. Each sample was cold pressed at $700 \mathrm{Mpa}$ in a rectangular mold $(5 \times 10 \mathrm{~mm})$ made of special tool steel. Which gave about $95 \%$ of the theoretical density. Hot pressing was carried out at $30 \mathrm{Mpa}$ in a vacuum furnace in which the pressure was maintained under $10^{-4}$ torr and temperature between 400 and $585^{\circ} \mathrm{C}$. The heating rate was $25^{\circ} \mathrm{C} \mathrm{min}{ }^{-1}$ and the hot pressing time was $30 \mathrm{~min}$ unless described otherwise. Thermoelectric properties were measured using specimens of $5 \times$ $5 \times 10\left(\mathrm{~mm}^{3}\right)$. The electrical resistivity $(\rho)$ was measured by the four probe method. The Seebeck coefficient $(\alpha)$ was obtained by measuring voltage between both ends of specimen after applying constant temperature difference between those ends. The thermal conductivity $(\kappa)$ was determined by the Harman $\operatorname{method}[4]$. The figure-of-merit $(Z)$ was determined using the 
relationship $\mathrm{Z}=\alpha^{2} / \rho \kappa$. The presence of oxides was checked by the XRD analysis.

\section{Result and discussion}

1. Thermoelectric properties of zone melted materials.

The composition for the zone melting growth was determined as $90 \% \mathrm{Bi}_{2} \mathrm{Te}_{3}-10 \% \mathrm{Bi}_{2} \mathrm{Se}_{3}$ which has low lattice thermal conductivity and large material factor $B[5]$. As grown ingots showed p-type properties. $\mathrm{CdCl}_{2}$ was used as a donor dopant to convert electrical

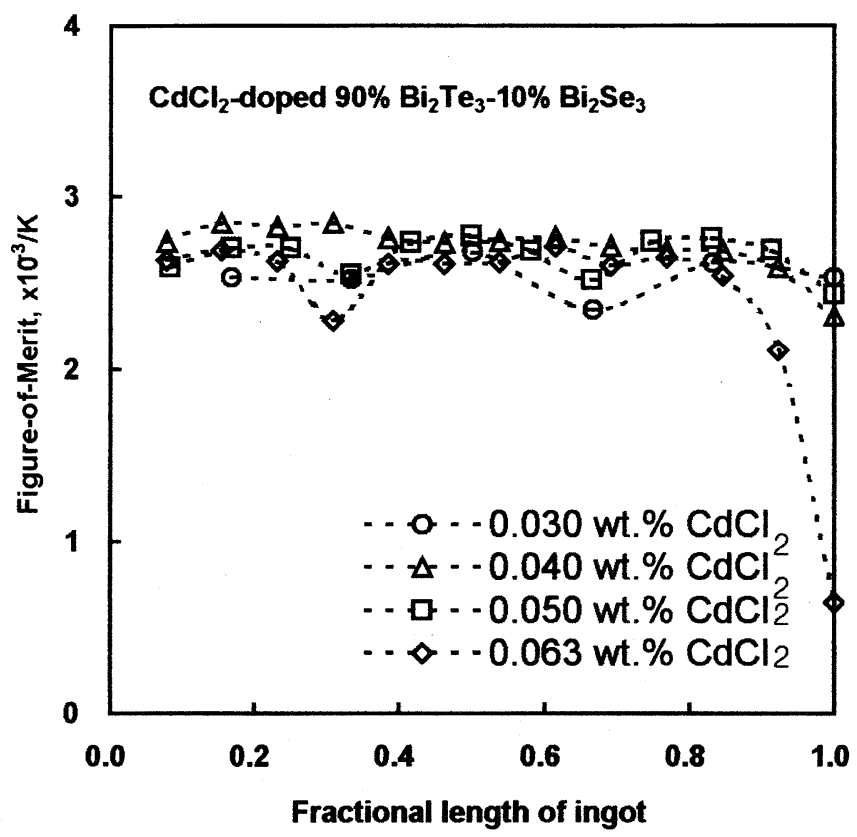

Figure 1. Variation of the Seebeck coefficient of zone melted ingots with different amount of dopant.

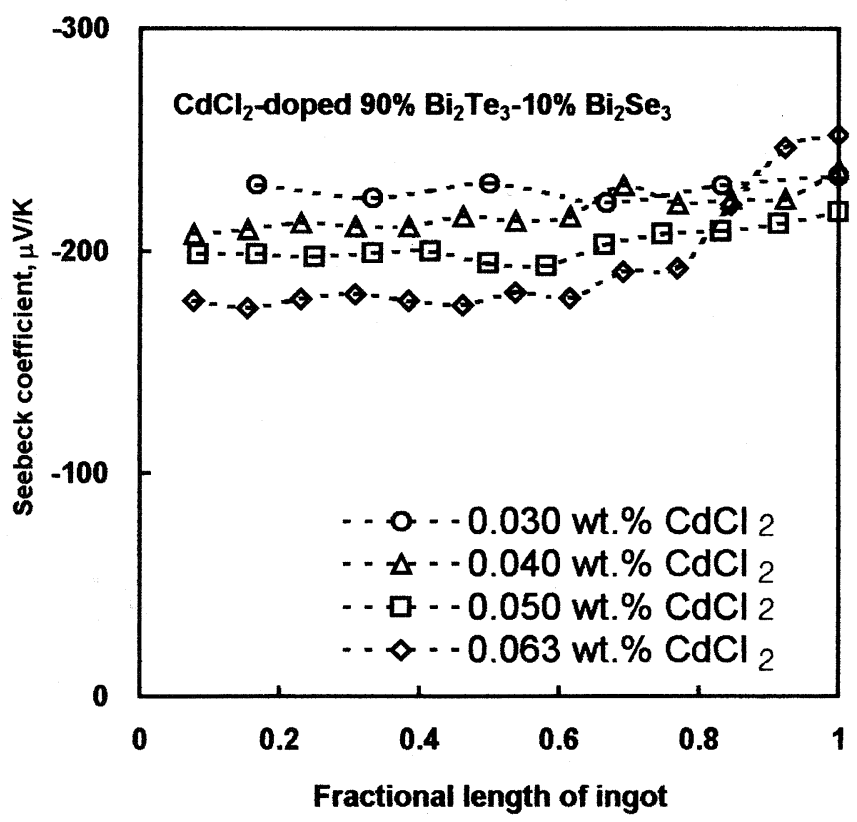

Figure 2. Variation of the electrical resistivity of zone melted ingots with different amount of dopant. type to n-type. As shown in Figure 1 The Seebeck coefficient of a zone melted ingot showed uniform values along the ingot except the end part of the ingot. The relationship between the Seebeck coefficient and electrical resistivity showed that this material was extrinsic when $\mathrm{CdCl}_{2}$ was added more than $0.03 \mathrm{wt} \%$. The decrease of the Seebeck coefficient with the increase of $\mathrm{CdCl}_{2}$ content, therefore, implies that electron concentration increases with the increase of $\mathrm{CdCl}_{2}$ content. The Figure of merit was also uniform along ingot. Compositional segregation was checked by the chemical analysis. There was considerable macro-segregation in composition along ingot because growth speed was slow $(0.1$ $\mathrm{mm} / \mathrm{min}$.). As shown in Figure 3 the composition of Se changed from $4 \mathrm{wt} \%$ to $1 \mathrm{wt} \%$ along the ingot. Rather uniform thermoelectric properties along the ingot in spite of considerable macrosegregation indicates that the compositional segregation affects little on the thermoelectric properties when Se content is less than $4 \mathrm{wt} \%$. In conclusion, thermoelectric properties can be maximized by optimization of the amounts of dopant when the ingot composition is fixed. The highest figure of merit, $\sim 3 \times 10^{-3} \mathrm{~K}^{-1}$, was obtained when $0.4 \% \mathrm{CdCl}_{2}$ was doped.

2. Thermoelectric properties of hot pressed materials.

\section{2-1. Effects of the mechanical deformation.}

It has been known that a defect introduced during the mechanical deformation of the $\mathrm{Bi}_{2} \mathrm{Te}_{3}-\mathrm{Sb}_{2} \mathrm{Te}_{3}$ system acts as a donor[6]. In the present study, the effect of deformation was investigated for the $90 \% \mathrm{Bi}_{2} \mathrm{Te}_{3}-10 \% \mathrm{Bi}_{2} \mathrm{Se}_{3}$ system. A specimen of $5 \times 5 \times 10\left(\mathrm{~mm}^{3}\right)$ was cut from a quenched ingot and cold pressed repeatedly within a mold. The degree of the mechanical deformation was quantified by counting the number of cold pressing. The variation of the Seebeck coefficient for cold pressed specimens was denoted as filled circles in Figure 4. An ingot of as grown $90 \% \mathrm{Bi}_{2} \mathrm{Te}_{3}-10 \% \mathrm{Bi}_{2} \mathrm{Se}_{3}$ solid solution was p-type whose Seebeck coefficient was about $100 \mu \mathrm{V} / \mathrm{K}$. With increasing the

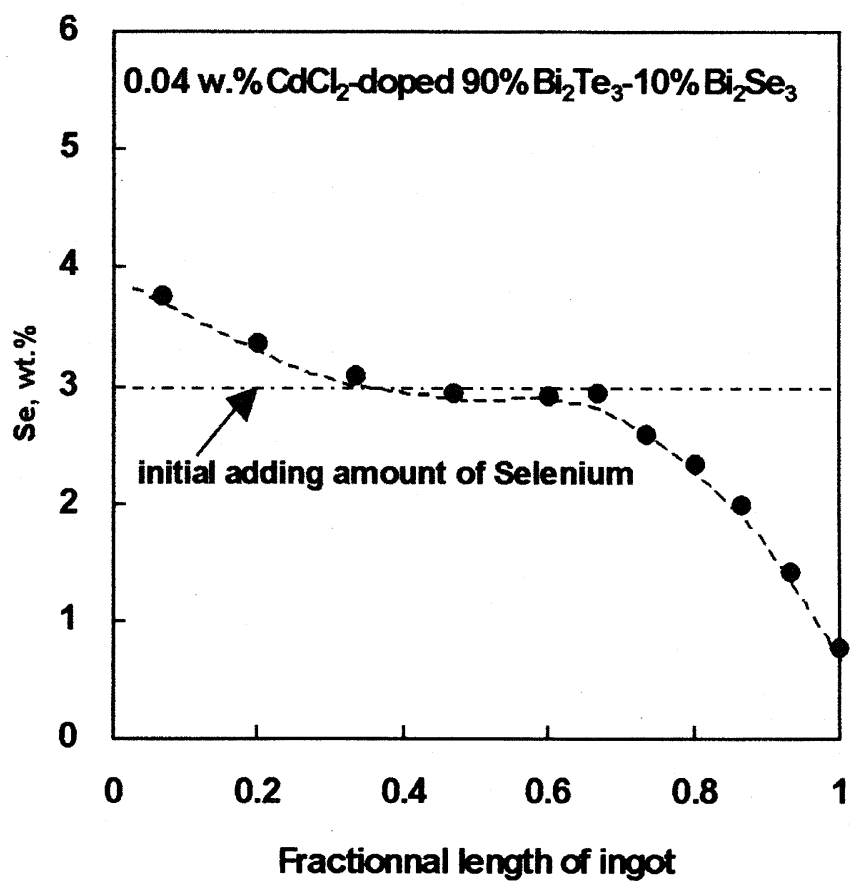

Figure 3. Macro-segregation of the composition of Se along an ingot. 
number of cold pressing, the Seebeck coefficient changed to negative value. This means that mechanical deformation in the $90 \% \mathrm{Bi}_{2} \mathrm{Te}_{3}-10 \% \mathrm{Bi}_{2} \mathrm{Se}_{3}$ solid solution also produces n-type carriers as in the $\mathrm{Bi}_{2} \mathrm{Te}_{3}-\mathrm{Sb}_{2} \mathrm{Te}_{3}$ system. It seems that no more carriers are generated after 3 times of repeated cold pressing and it reaches a saturation state. Unusually low electrical conductivities of pressed specimens indicates that the amount of generated carriers is not very much because the Seebeck coefficient changed just within the intrinsic range where the electrical resistivity is high due to low carrier concentration. The variation of the Seebeck coefficient after hot pressing of cold pressed specimens at $500^{\circ} \mathrm{C}$ for half an hour was denoted as open circles in Figure 4. Combined data of

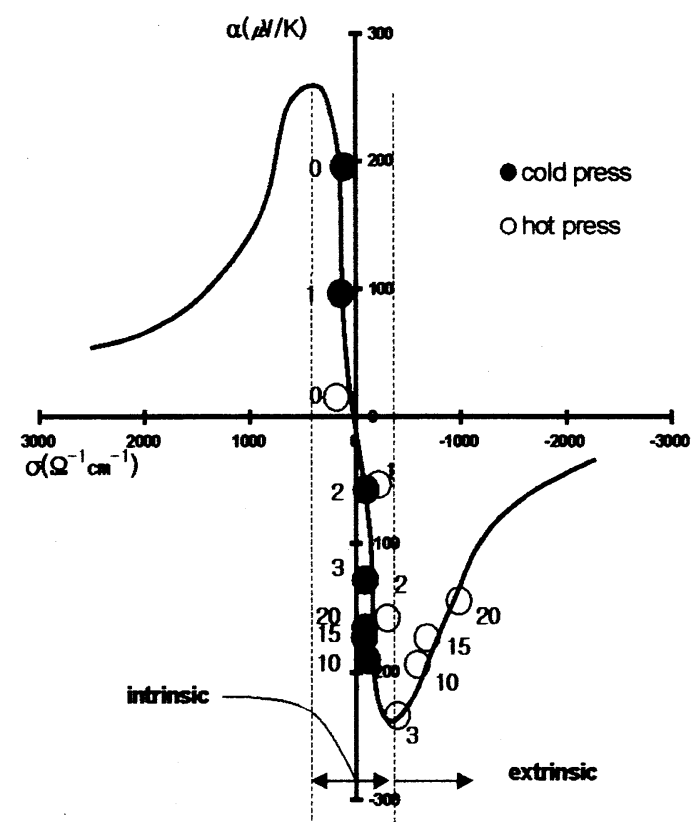

Figure 4. Change of the Seebeck coefficient and electrical conductivity for repeatedly cold pressed and hot pressed specimens as a function of the number of the cold pressing.

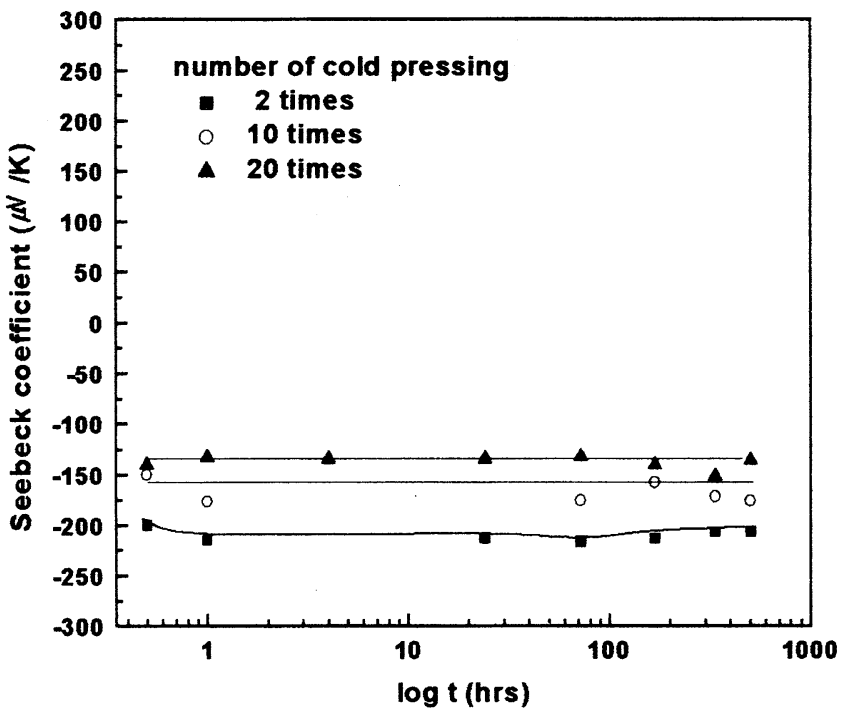

Figure 5. Effect of annealing on the Seebeck coefficient of specimens undergone different times of cold pressing. cold pressed and hot pressed form a typical Jonker plot[7]. Area in the Jonker plot within dotted lines indicates that materials are intrinsic when the Seebeck coefficient and the electrical conductivity lie within this area. Points undergone hot pressing located outside of this region on the plot. This means that specimens cold pressed more than three times become extrinsic when they are hot pressed. With the increase of the number of cold pressing, the negative Seebeck coefficient in magnitude is decreased in the extrinsic range and the electrical conductivity also increases. This means that more n-type carriers are generated with the increase of the number of cold pressing when they are hot pressed. If we compare the variations of the Seebeck coefficient for specimens cold pressed 3-20 times, as the number of cold pressing increases the Seebeck coefficient changes further after hot pressing although their initial Seebeck coefficients at cold pressed state were similar. This means that the degree of the mechanical deformation rather than initial carrier concentration dominantly affects on the final state of hot pressed specimens. One can speculate the reason for this result as two directions. The first reasoning for this result would be that the difference of diffusion rate which depends on the degree of previously received mechanical deformation resulted in different properties. If this assumption is right, prolonged hot pressing time should render the Seebeck coefficient of hot pressed samples to have same value. The other reasoning would be such an argument that different amount of previously received mechanical deformation caused their specimens to reach different meta-stable state. To clarify what is more plausible reason, cold pressed samples with different pressing number were annealed at $500^{\circ} \mathrm{C}$. Figure 5 shows the variation of the Seebeck coefficient as a function of cumulative annealing time. The Seebeck coefficient of the sample cold pressed two times increased and then saturated with increase of the annealing time. Initial increase of the Seebeck coefficient for the sample cold pressed twice was within intrinsic range. Therefore variation of carrier concentration was small. Meanwhile, The Seebeck coefficient of samples cold pressed more than three times was extrinsic when they were annealed and does not show noticeable change with annealing time. The Seebeck coefficient

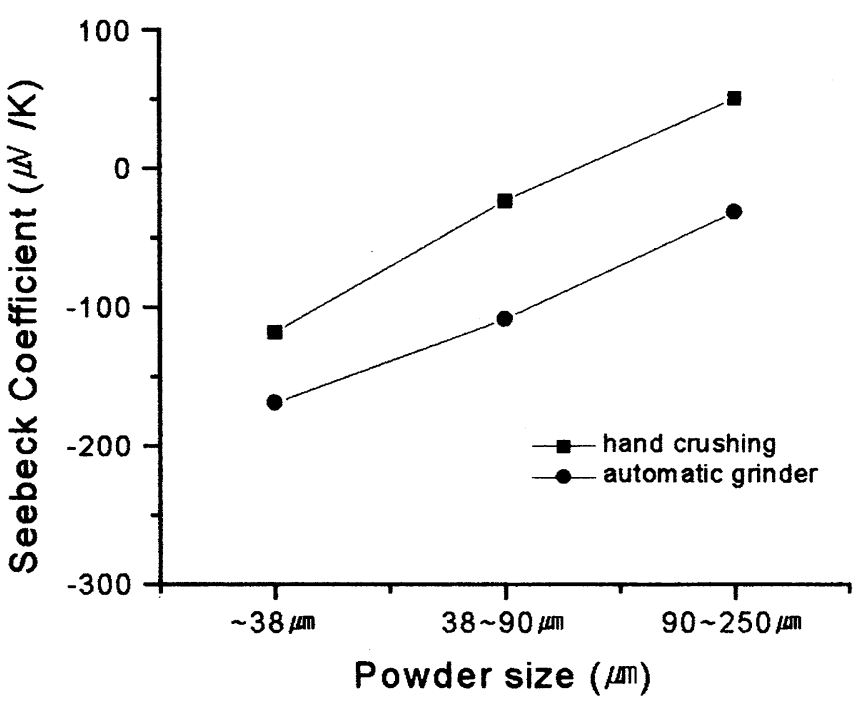

Figure 6. Effect of pulverizing method on the Seebeck coefficient of cold pressed specimens. 
were different depending on the number of cold pressing and the difference was preserved even when annealed for 7 days at $500{ }^{\circ} \mathrm{C}$. This suggests that for the explanation of the above experimental result the second reason is plausible as long as samples were annealed at below $500^{\circ} \mathrm{C}$.

\section{2-2. Effects of the particle size and pulverizing method}

As the degree of the mechanical deformation affects thermoelectric properties of materials, we expected that the particle size as well as pulverizing method could affect on the thermoelectric properties of hot pressed samples. The Seebeck coefficient of powders prepared by hand crushing and automatic grinding were compared in Figure 6. At a fixed particle size, the Seebeck coefficient of mechanically grinded specimen shows larger value in magnitude than that of hand crushed. This result agrees well with the results obtained from the effects of the mechanical deformation if powers grinded by automativ grinder received more deformation compared to powders crushed with hands. From the electrical resistivity measurement, it was found that all specimens were intrinsic. The Seebeck coefficient of small size particle is larger in magnitude than that of large size particle regardless pulverizing method. This result is also understandable because small size particles will have chances to experience more mechanical deformation.

\section{2-3. Effects of oxidation.}

It was well known that oxygen act as a donor when it is in bismuth telluride based solid solutions. In the course of the hot pressing process, powders are exposed to air during pulverization. There could be also a possibility of accidental exposure to air during handling at high temperature. In order to examine the influences of oxidation, one kind of powders was intentionally exposed 4 hours in the air at different temperatures and the other

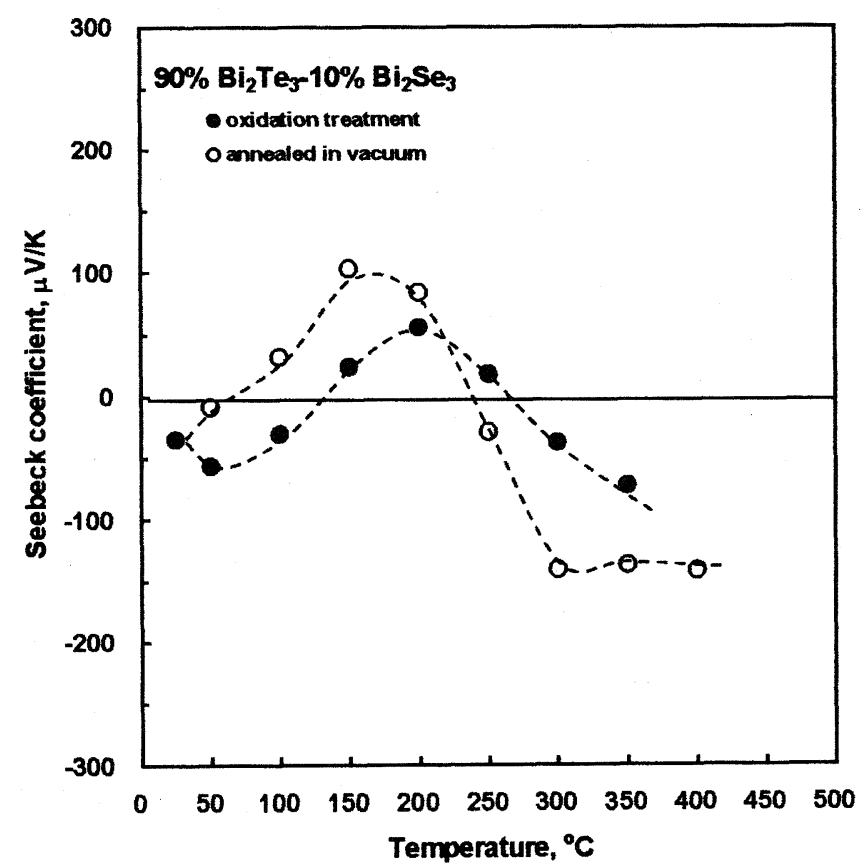

Figure 7. Variation of the Seebeck coefficient of specimens; opened circles annealed at oen air and filled circles annealed under a vacuum. powders were kept at the same condition under a vacuum and then their thermoelectric properties were compared after compacting powders into rectangular specimens. Figure 4 shows the variation of the Seebeck coefficient of specimen as a function of annealed temperature. One can see that the variation trend of their Seebeck

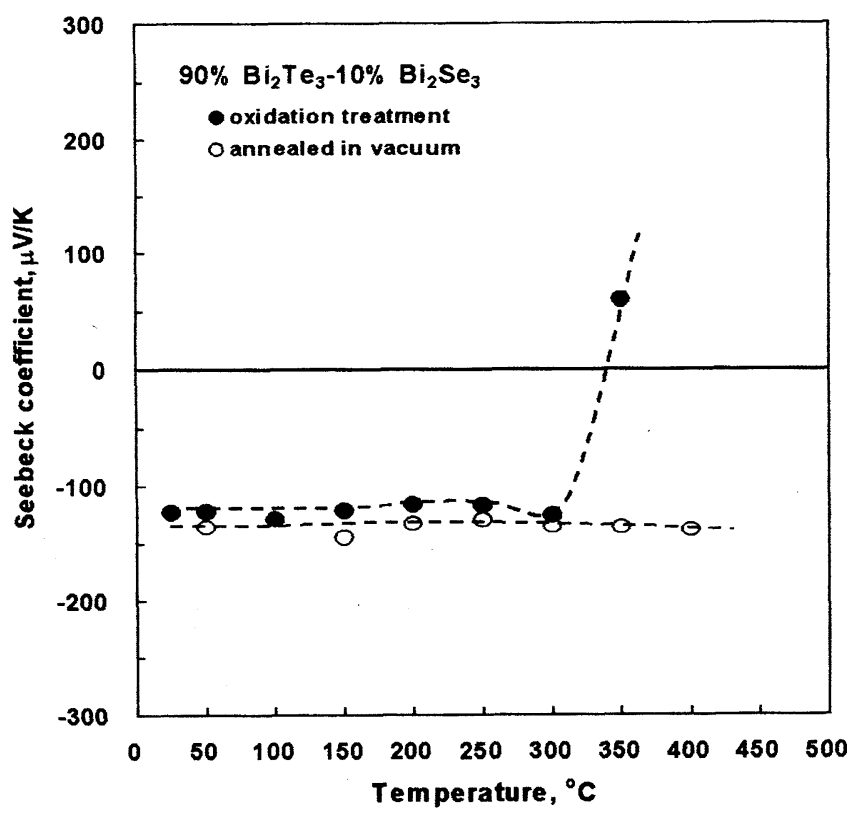

Figure 8. Variation of the Seebeck coefficient of specimens; opened circles annealed at oen air and filled circles annealed under a vacuum.

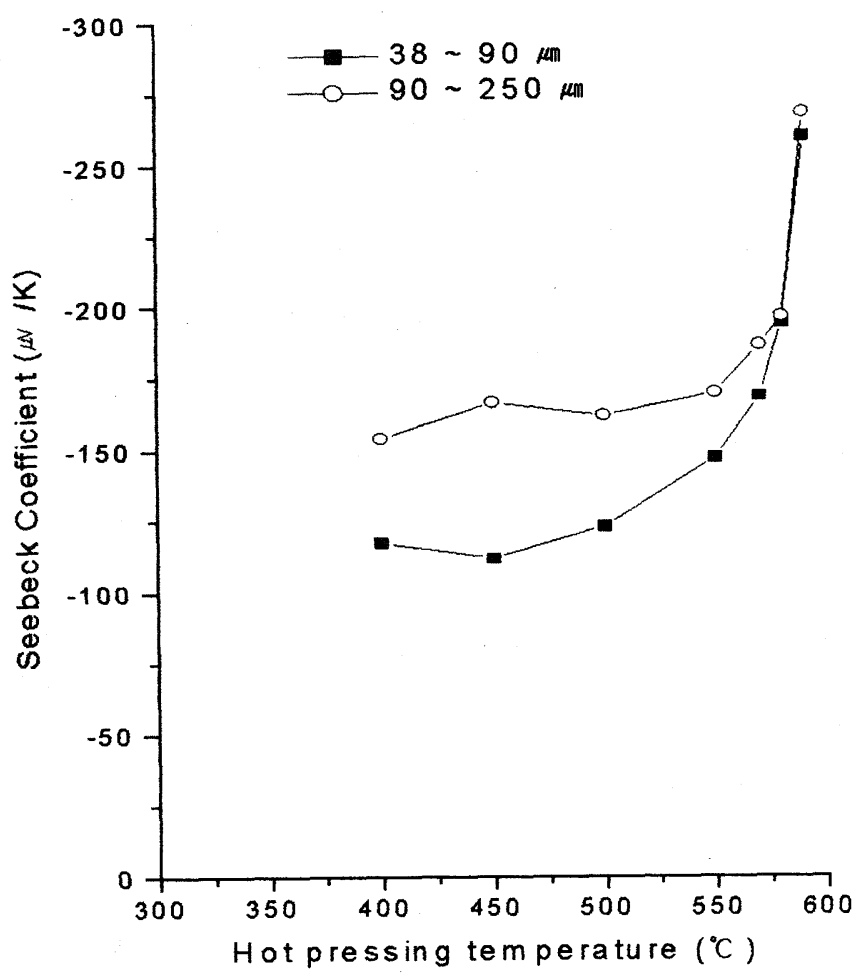

Figure 9. Variation of the Seebeck coefficient with hot pressing temperature for powders with different particle sizes. 
coefficient is same although the absolute values have some difference. With increasing annealing temperature the Seebeck coefficient changed from negative to positive value and it returned again to negative at high temperature. The Seebeck coefficient of the sample exposed to air at $400^{\circ} \mathrm{C}$ could not be measured because of severe oxidation. The seebeck coefficient of specimens in Figure 4 seems to converge at high annealing temperature regardless of oxidation treatment. To check the convergence, specimens annealed at different temperature were hot pressed at $500{ }^{\circ} \mathrm{C}$. Figure 5 shows that al specimen except a specimen annealed at $350^{\circ} \mathrm{C}$ in air show slight small value compared to those of annealed in a vacuum. Interesting point is that the Seebeck coefficient of hot pressed samples does not change with preannealing temperature. Which suggest that there is certain degree of oxidation through exposure of powders to open air. This oxide diffuses into matrix during hot pressing and increases the carrier concentration of matrix to a certain extent. Further oxidation at higher temperature seems not to affect on the carrier concentration. This means that the solubility of oxygen in the matrix is not very high and it is quickly attained. This result agrees well with results of Tsipin at al [8]. They reported that solubility of oxygen in the bismuth telluride was about 0.01 at \%. The Seebeck coefficient of a specimen annealed at $350^{\circ} \mathrm{C}$ changed negative to positive value. It seems that this is related with the formation of oxide because oxide peak appeared in the XRD analysis of specimens annealed at higher than $350^{\circ} \mathrm{C}$.

\section{Effects of the hot pressing temperature.}

Powders with different particle size have different values of Seebeck coefficient as shown in Figure 3. These powders were hot pressed at various temperatures. As shown in Figure 6, The Seebeck coefficients for specimens hot pressed at below $500{ }^{\circ} \mathrm{C}$ show difference in the Seebeck coefficient for different particle size. This result agrees well with result of Figure 1 and Figure 2 where the annealed specimens which received more mechanical deformation previously have small Seebeck coefficients compared to those received less deformation. It is plausible to think that powders with small particle size experiences more mechanical deformation than those of large particle size. In other words, n-type carriers of specimens which received more mechanical deformation (small size particle) increases more compared to specimens which received less deformation when they are hot pressed. On the other hand, the Seebeck coefficient of hot pressed samples at temperature than $550^{\circ} \mathrm{C}$ seems to converge to nearly same values regardless particle size. This means that mechanical deformation does not affect on the carrier concentration of hot pressed specimen when the hot pressing temperature become close to the melting point of the alloy. At the moment, it is not clear which type of defect was created when a specimen had been received mechanical deformation was hot pressed. Further studies are required to clarify the characteristic of the defect.

\section{Conclusions}

Alloys of the $\mathrm{Bi}_{2} \mathrm{Te}_{3}$ rich side of $\mathrm{Bi}_{2} \mathrm{Te}_{3}-\mathrm{Bi}_{2} \mathrm{Se}_{3}$ were prepared by the zone melting method and the hot pressing method and results of the study on their thermoelectric properties can be summarized as follows. Thermoelctric properties of zone melted ingot were largely affected by dopant when the ingot composition is fixed, wheras thermoelectric properties of hot pressed material were mainly related with variation of the carrier concentrations caused by the generation of electrically active defects from many sources. The compositional segeregation along the zone melted ingot affect little on the thermoelectric properties when Se content was less than $4 \mathrm{wt} \%$. The highest figure of merit, $3 \times 10^{-1} \mathrm{~K}^{-1}$, was obtained when $0.4 \mathrm{wt} \% \mathrm{cdcl}_{2}$ was doped to $90 \% \mathrm{Bi}_{2} \mathrm{Te}_{3}-10 \% \mathrm{Bi}_{2} \mathrm{Se}_{3}$ Defects induced by the mechanical deformation and oxygen causes the generation of donors. Defect concentration is also altered with different hot pressing temperatures. The surface oxidation generates n-type carrier, but the amount was not large to considerably affect on the carrier concentration of the hot pressed sample. The mechanical deformation also generates small amounts of carriers, but this cause hot pressed specimens to become extrinsic due to the generation of large number of n-type carriers.

\section{REFERENCES}

1) Kokosh G. V. and Sinai S. S., Soviet Phys. Sol. State 2, (1961) pp 1012-1018.

2) Tokiai T., Uesugi T. and Yoneyama Y., in Proceedings of the $12^{\text {th }}$ International Conference on Thermoelectrics, Yokohama Japan (Edited by K. Matsuura) (1993) pp 281-285.

3) Shin H. S., Ha H. P., Hyun D. B., Shim J. D. and Lee D. H. J. Phys. Chem. Solids 58 No4, (1997) pp 671-678.

4) Harman T. C., Cahn J. H. and Logan M. J., J. Appl. Phys. 30 (1959) pp 1351-1359.

5) Ha H. P., Cho Y. W., Hyun D. B. and Shim J. D.., in Proceedings of the $13^{\text {th }}$ International Conference on Thermoelectrics, Kansas U. S. A. (Edited by Mathiprakasam B.) (1994) pp 235-238.

6) Schultz J. M., McHugh J. P. and Yiller W. A., J. Appl. Phys. (1962) pp 2443-2450.

7) Jonker G. H., "The Application of Combined Conductivity and Seebeck Effect for the Analysis of Semiconductor Properties", Philips. Res. Rept., 23 (1968) pp 104-134.

8) M. I. Tsipin and L. T. Evdokimenko, IZV. AN SSSP. Neorganicheskie Materiali 9 No4 (1973) pp 580-586. 